\title{
La Personalización de la Intervención Educativa Proporciona Igualdad de Oportunidades a los Menores en Situación de Riesgo
}

\author{
Personalising Educational Intervention Provides Equality of \\ Opportunities to Under Eighteen at Risk
}

\section{Intervenção personalização Educacional Fornece Igualdade de Oportunidades para Crianças em Risco}

David Herrera-Pastor * José Manuel de-Oña-Cots

Universidad de Málaga

\begin{abstract}
Este artículo se desprende de la investigación llevada a cabo en torno a la historia de Suleimán, un adolescente marroquí que viviendo en situación de pobreza decidió migrar irregularmente a España para buscar otra vida. Fue arrestado por traficar con drogas antes de cumplir la mayoría de edad, fue sentenciado a un proceso de Reforma Juvenil de dos años. Lo interesante de su caso fue el giro positivo que dio al experimentar dicho proceso, pues pasó de "Menor Infractor" a "Educador de Menores" gracias, fundamentalmente, a aquel proceso de reforma. El método biográfico fue la metodología de investigación empleada. Una de las claves que emergieron fue la personalización de la intervención. Intervención que, sustentada en distintos elementos, permitió a Suleimán transformar la situación determinista que vivía y encauzar su vida de la manera que él quería. En las conclusiones se reivindica, fundamentalmente, la necesidad de personalizar los procesos de intervención con menores en situación de riesgo, y contribuir en su desarrollo personal y social.
\end{abstract}

Descriptores: Igualdad de oportunidades, Educación, Justicia social, Joven desfavoredido.

This paper comes from a research developed around Suleimán's history, a Moroccan adolescent who lived in poverty and decided to migrate illegally to Spain, because he wanted to look for a better life. He was arrested (being under eighteen) for drug trafficking, he was sentenced to two years' rehabilitation process. His case is interesting because it turned in a positive way, from being a "Young Offender" he became an "Educator of Minors", essentially, because of the mention process. The biographical method was the research methodology used. One of the keys which emerged from the analysis was the personalisation of the intervention. The intervention, based on different elements, provided Suleimán the chance of transforming the deterministic situation he lived and conduct his life how he wanted. Essentially, the conclusions claim the necessity of personalising the intervention processes with under eighteen at risk and contribute in their personal and social development.

Keywords: Equality of opportunity, Education, Social justice, Disadvantaged youth.

*Contacto: dvherrera@uma.es

ISSN: 2254-3139

www.rinace.net/riejs/

revistas.uam.es/riejs
Recibido: $\quad 15$ de diciembre 2016

$1^{\text {a }}$ Evaluación: 23 de enero 2017

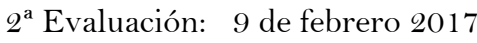

Aceptado: $\quad 6$ de marzo 2017 


\begin{abstract}
Este artigo resulta de uma investigação desenvolvida em torno da história de Suleiman, um adolescente marroquino que, vivendo na pobreza, decidiu emigrar ilegalmente para a Espanha para encontrar uma outra vida. Suleiman foi preso por tráfico de drogas antes de atingir a maior idade e foi condenado a um processo de recuperação juvenil de dois anos. A coisa interessante sobre o caso foi o resultado positivo que deu a experiência desse processo, uma vez que passou de "joven delinquente" para "Educador de menores", principalmente graças ao processo de recuperação. O método biográfico foi a metodologia de pesquisa utilizada. Uma das chaves que emergiu foi a personalização da intervenção. Intervenção que, com base em diferentes elementos, permitiu a Sulayman transformar a situação determinista em que vivia para a vida que ele queria. Nas conclusões é reivindicada a necessidade de personalizar o processo de intervenção com crianças em risco, e de contribuir para o seu desenvolvimento pessoal e social.
\end{abstract}

Palavras-chave: Igualdade de oportunidades, Educação, Justiça social, Jovens desfavorecidos.

Este artículo se desprende del trabajo de investigación (Tesis) subvencionado por la Junta de Andalucía en el marco de la (beca de investigación) "Ayuda para la Formación de Docentes e Investigadores de las Universidades Andaluzas" (2004-2008), de la que fue adjudicatario el primer autor del artículo.

\title{
Introducción
}

En este artículo se analiza uno de los aspectos que resultaron fundamentales en la evolución de la historia de Suleimán, un joven marroquí nacido en el seno de una familia numerosa (10 hijos) que disponía de muy pocos recursos económicos y cuyas condiciones de vida eran duras: su casa no tenía agua; su pequeña aldea se ubicaba a las afueras de la ciudad y se encontraba un tanto aislada (entre otras cosas, porque estaba mal comunicada: la carretera no estaba asfaltada y tenía socavones). A los 13 años abandonó la escuela para contribuir con la economía familiar. Trabajaba jornadas de aproximadamente 12 horas en una cafetería para ganar 10 dírhams al día (el equivalente a un euro). Él, que era el menor de sus hermanos, sentía que su futuro estaba prácticamente escrito (casi todos sus hermanos varones eran conductores de camión) y no quería conformarse con la vida a la que parecía estar predestinado.

Tras errar durante varios años por distintos trabajos precarios decidió migrar a España de manera irregular para tratar de buscar un futuro mejor. Estando allí, y siendo menor de edad, fue detenido por cometer un delito de tráfico de estupefacientes. Como consecuencia, el Sistema de Justicia Juvenil le impuso un proceso de reforma de dos años, que se dividió en dos medidas: la primera, Internamiento en centro (en régimen semiabierto) y, la segunda, Libertad vigilada. Finalizando esta última comenzó a trabajar en un piso tutelado de menores en situación de protección, al principio como monitor y después como educador. Allí lleva trabajando desde hace más de diez años.

Uno de los motivos principales que animaron la puesta en marcha de la investigación fue la evolución del caso: el protagonista de la historia pasó de "menor infractor" a "educador de menores" y parecía que su proceso de reforma juvenil había resultado fundamental en ello. El foco de investigación se concretó en tratar de vislumbrar cuáles fueron las claves que habían contribuido a que la historia se desarrollara como lo hizo, y poder analizarlas. $\mathrm{Y}$, al mismo tiempo, se quería conocer qué había tenido de "educativo" todo aquel proceso. 
Para poder localizar todas las claves y examinar el carácter pedagógico de dicho proceso resultaba necesario conocer la historia de Suleimán de manera completa, se necesitaba tener una panorámica de su vida para comprender la trascendencia que tuvo el proceso de reforma en su trayectoria vital. Ése fue uno de los principales motivos por los que se eligió el método biográfico para llevar a cabo la investigación.

Para construir la historia de vida se acudió a numerosas fuentes de información y se utilizaron diversas técnicas de recogida de datos. Se entrevistó a Suleimán (en numerosas ocasiones) y a diecisiete personajes que fueron significativos en el desarrollo de su historia: la jueza de menores, el subdirector del Centro del Internamiento de Menores Infractores (CIMI), su tutor en el CIMI, la tutora de Libertad Vigilada, su madre de acogida, su hermano biológico, etc. Además, se tuvo a acceso a numerosos documentos de carácter oficial (la sentencia, evaluaciones, informes de evolución, etc.) y se consiguió un registro iconográfico que ilustraba muchas de sus vivencias. La mayoría de fotografías recogían experiencias vividas durante la medida de internamiento en centro. Se utilizaron también otras técnicas y se recabaron otros datos. En definitiva, se recabó un compendio de información cuantiosa que fue cruzada correspondientemente para elaborar la biografía de manera exhaustiva, rigurosa y coral. Siendo ésta construida de acuerdo al modelo de relatos cruzados, de manera no consecutiva.

En este artículo se va a analizar una de las claves que resultaron fundamentales para que el caso de Suleimán evolucionase favorablemente: la personalización de la intervención. Llevar a cabo aquella intervención atendiendo a las características singulares del educando y contribuyendo en la satisfacción de las distintas necesidades que se planteaban fue esencial para transformar su situación y que nuestro protagonista pudiera re-encauzar su vida. En virtud de ello, se puede decir que su caso comprende una historia de justicia social, ya que a través de su proceso de reforma se consiguieron contrarrestar todos aquellos factores de riesgo que le condenaban a una situación pobreza, irregularidad, delincuencia y tuvo la oportunidad de construir una vida que le satisfacía, alejado de todo ello.

A partir del análisis realizado, la idea más significativa que se saca en claro es que para optimizar la intervención educativa con menores en situación de riesgo hay que procurar la personalización de los procesos. Y para que eso ocurra, además de intentar suscitar en el educando los aprendizajes que se consideren oportunos, se ha de contribuir en la satisfacción de las diversas necesidades que plantee cada caso. Por eso el programa de intervención debe ser pedagógico, pero (como mínimo) también social.

\section{Fundamentación teórica}

En este apartado queremos revisar los presupuestos teóricos en los que se apoya la idea sobre la que gira este trabajo: la personalización de la intervención pedagógica que se plantea con menores en situación de riesgo. Dicha revisión se divide en dos epígrafes: por un lado, se explicitan las características básicas que ha de poseer una intervención personalizada. Y por otro, sostenemos que para intervenir pedagógicamente con este colectivo, además de educar, hay que contribuir en la satisfacción de las necesidades que plantee cada caso, ya que si no se trabaja en ese sentido, el proceso pedagógico se puede ver lastrado. En relación a esto, se realiza una revisión concisa de la Teoría de las Necesidades Humanas de Maslow. 


\subsection{Características básicas del programa de intervención personalizado}

En el ámbito de los menores en situación de riesgo el programa de intervención que se quiera llevar a cabo debe tener un carácter personalizado. Tratar de realizar un programa lo más individualizado posible (Longás y Riera, 2011) debe ser un rasgo fundamental dentro de cualquier actividad que pretenda ser educativa. Algunas de las características básicas que ha de poseer esta individualización pueden ser, entre otras:

Por un lado, debe ser 'único' porque cada caso es único, ya que está inmerso en una situación singular. Cada ser humano es irrepetible, con potencialidades y capacidades propias que posibilitan la construcción de una determinada forma de hacer y relacionarse con los demás. Se debe personalizar el programa, diseñarlo y materializarlo a partir de las características genuinas de cada caso (Trilla, Gros, López y Martín, 2011). La puesta en marcha de planteamientos homogéneos que atiendan a todos los menores por igual, difícilmente podrá dar respuesta a las necesidades particulares que cada caso requiera.

Además, no se debe realizar la intervención a partir de la acumulación de acciones (Naranjo, 2007), mucho menos si éstas se llevan a cabo de manera descoordinada e inconexa. Las iniciativas aisladas no ayudan a abordar la problemática en toda su complejidad. Como dice Vega (1994, p. 117) "de poco servirán las respuestas puntuales, si no existe una respuesta global a todas las cuestiones que la problemática (...) plantea”.

Por otro lado, ha de ser 'integral', en cuanto que ha de atender los diversos elementos que conforman cada caso. La intervención debe atender los aspectos pedagógicos que se considere, pero también debe tratar de ayudar al educando a dar respuesta a todos aquellos aspectos que necesiten ser atendidos porque pudieran estar condicionando su desarrollo (por ejemplo, aspectos fisiológicos, afectivos, psicológicos, etc.). En muchos casos, la desatención de alguno de esos aspectos puede impedir la adecuada evolución de la intervención, incluso echarla abajo. Abordar todos los problemas que se encuentran en cada caso no garantiza la consecución de los objetivos propuestos, pero no preocuparse por ellos dificulta su progreso. A realidad compleja, programa complejo (Sánchez-Raya y Alonso García, 2013, p. 83).

Desde el programa de intervención se han de tratar, por tanto, cuantos elementos sean necesarios, en virtud de las necesidades singulares de cada sujeto. La clave de la personalización estará en la cobertura de las distintas necesidades y en el tipo de interacción que se establezca entre los distintos elementos que se conjuguen en cada programa.

En ese sentido, los profesionales deben buscar estrategias que les permitan conocer a cada persona con la que se ha de intervenir de manera global y exhaustiva. Atender, únicamente, facetas o aspectos parciales del sujeto, difícilmente proporcionará su desarrollo integral: "La educación integral favorece y pone a disposición del educando todo lo necesario para conseguir su grado óptimo de madurez como persona y como miembro de la sociedad" (Sánchez-Raya y Alonso García, 2013, pp. 119-120).

Y, por último, el programa debe tener un carácter 'participativo', en cuanto que debe involucrar al educando en su desarrollo, para que sea él mismo quien pueda construir a partir de la experiencia pedagógica. Que las personas participen de sus procesos evolutivos es condición indispensable para que se desarrollen de manera armónica y holística. Se habrán de poner en juego, por tanto, las estrategias correspondientes para que la persona se involucre en el proceso de manera activa, generando espacios de 
corresponsabilidad y cooperación (Gichot, 2013). Si el individuo participa de esa manera, el programa podrá tener mayores posibilidades de éxito. Por eso la acción pedagógica no ha de plantearse sobre el sujeto, sino con el sujeto (Esteve, 2010). Conseguir que esa implicación se suscite es el reto de los profesionales que materializan el programa.

\subsection{Para personalizar la intervención, además de educar, hay que contribuir en la satisfacción de las necesidades que plantee cada caso}

Muchos de los aspectos que se satisficieron en el caso sujeto a estudio, a priori no tenían una relación directa con los objetivos educativos propuestos. Sin embargo, resultaron fundamentales para que el caso evolucionara como lo hizo.

Tal como dice Subirats (en Alonso, Ruiz, Sánchez y Oficialdegui, 2014) existen una serie de aspectos (alimentación, vivienda, ingresos, salud, entorno social, etc.), que resulta necesario satisfacer en los procesos de desarrollo, porque de lo contrario se pueden convertir en factores de riesgo. En la biografía de Suleimán todos los aspectos circunstanciales que se consideraron necesarios fueron satisfechos durante el proceso de reforma juvenil y, según los datos, haberlos satisfecho fue una de las claves principales para que su historia diese aquel giro.

Tratar de llevar a cabo un proceso pedagógico sin atender a las circunstancias que rodean el caso, difícilmente permitirá alcanzar los objetivos propuestos. Por eso, cuando se actúa educativamente con menores en situación de riesgo, el programa de intervención debe ser pedagógico, pero también, y como mínimo, social. Lo pedagógico y lo social no se puede separar. No se debe desarrollar un programa de intervención sin tener en cuenta ambos aspectos.

Hay que tener presente que, por encima de todo, el ser humano es un ser social y su configuración como tal está mediada por las características de las realidades de las que forma parte. Lo social es parte intrínseca de lo humano.

A veces, se trabaja en el ámbito socio-pedagógico deshumanizando el proceso, sin atender a lo que hace humano al 'ser humano', que son las circunstancias socio-culturales que le rodean. La educación no se puede desarrollar de manera abstracta, tratando a la persona sin prestar atención a las características de su entorno. Por tanto, se ha de procurar el desarrollo del sujeto teniendo en cuenta sus características singulares, pero también las características del contexto en el que se encuentra inmerso.

Cuando hablamos del colectivo de menores, ya sea en el ámbito de Reforma Juvenil, de Protección o de cualquier otra índole, se ha de hacer un mayor hincapié sobre esta idea por dos motivos: 1) porque el educando se encuentra en una edad evolutiva trascendental y 2) porque intervenir adecuadamente suele resultar necesario y determinante para que el sujeto se desarrolle de forma correcta desde un punto de vista personal y social.

Por eso, en los casos donde las circunstancias sociales resulten complejas la intervención se debe interpretar y poner en marcha desde un marco, como mínimo, Socio-Pedagógico. En ese sentido, la intervención no debe centrar su atención, únicamente, en la transformación del sujeto, sino que además de ella, debe contribuir en el establecimiento de una nueva configuración de sus circunstancias sociales (Luque y Lalueza, 2013). 
Algunos elementos que generalmente contribuyen a establecer esa nueva configuración son: disminuir los factores de riesgo (obstáculos para satisfacer a las necesidades básicas, dificultades relacionales, problemas de autoestima, etc.) y trabajar sobre los factores de protección (capacidad de establecer nuevos vínculos, establecimiento de redes sociales de apoyo, etc.), facilitando la creación de algunos nuevos y estimulando las potencialidades singulares del sujeto.

\subsubsection{Teoría de las Necesidades Humanas}

Teniendo en cuenta que las distintas necesidades que plantee cada caso pueden convertirse en factores de riesgo para el sujeto, entendemos necesario presentar una breve revisión de la teoría de las necesidades humanas, que puede ayudar a comprender cómo dichas necesidades pueden mediatizar el desarrollo de una persona.

A lo largo de la historia ha habido varios investigadores que han tratado de identificar las necesidades básicas que presenta todo ser humano. Probablemente, la teoría de las necesidades humanas que presentó Maslow (a mediados del siglo Xx) es la más conocida hasta la fecha. Este autor entendía que todas las personas poseían unas necesidades mínimas comunes. Según él, dichas necesidades se organizan de manera jerárquica y piramidal; en el sustrato más bajo se ubican las necesidades más elementales y en el más alto las que, con su satisfacción, contribuyen al desarrollo pleno. Lo estructuraba de esa manera porque entendía que el sujeto es un ser integral que cuando tiene una necesidad, dicha necesidad lo ocupa y lo domina. Así cuando un individuo está hambriento, el hambre es sentida por todo su ser, no sólo por su estómago. Y, como consecuencia, no sólo se alteran aquellos órganos que directamente se vinculan a la demanda, sino que lo hace todo él como persona: sus emociones, sus pensamientos, etc. Las necesidades se convierten, por tanto, en "determinantes activos u organizadores del comportamiento" (Maslow, 1975, p. 89), ya que tratan de poner a su servicio todas las potencialidades del sujeto para intentar satisfacer la necesidad que en ese momento acapara toda su atención.

A continuación, se presentan dichas necesidades en el orden (ascendente) que él las planteaba:

- En la base se sitúan las necesidades fisiológicas. "El ser humano que careciese de todo, tendería a satisfacer las necesidades fisiológicas antes que las otras" (Maslow, 1975, p. 86).

- En el segundo escalón se encuentran las necesidades de seguridad. Estar fuera de peligro, amenaza o riesgo es la principal demanda.

- Después están las necesidades afectivas y sociales. Que giran en torno a la necesidad de afiliación, de pertenencia, de sentirse parte de un grupo humano.

- Más adelante se ubican las necesidades de reconocimiento o de autoestima. No se trata, únicamente, de estar en el mundo, sino de que la existencia tenga algún valor. A través de esta necesidad se persiguen "sentimientos de autoconfianza, valía, fuerza, capacidad y suficiencia, de ser útil y necesario en el mundo" (Maslow, 1975, p. 95). La no satisfacción de estas necesidades puede derivar en: falta de confianza, inseguridad, indeterminación, sentimientos de inferioridad, etc. 
- En la cumbre de la pirámide se establecen las necesidades de autorrealización. Cada persona buscará satisfacer esta necesidad de manera singular (habrá quien la trate de satisfacer ayudando a los demás, preservando la naturaleza, a través del deporte, etc.).

Estas cinco categorías estaban divididas en dos niveles (inferior y superior): las necesidades más básicas serán las de nivel inferior y las de reconocimiento y autorrealización las de nivel superior.

\begin{abstract}
Maslow diferencia entre necesidades de nivel inferior y necesidades de nivel superior. Las primeras son fundamentales para el individuo y determinan un claro control sobre su conducta cuando no son satisfechas. Una vez satisfechas las necesidades básicas de subsistencia se entra en las necesidades sociales de autoestima y de pertenencia a un grupo. Tras la satisfacción de estas últimas se estará en disposición de cubrir las específicas de tipo intelectual y de autorrealización personal. (Sarramona, 2008, p. 114)
\end{abstract}

A pesar de ser una teoría vigente y seguida en la actualidad desde distintas áreas de conocimiento, ha recibido varias críticas a lo largo del tiempo. Las dos más destacas son: a) Por un lado, se pone en tela de juicio que elaboró su teoría en abstracto. Sus planteamientos no tienen una base empírica ya que nunca se examinaron de manera científica, es decir, no se sometieron a un escrutinio riguroso, siguiendo una metodología de investigación exhaustiva. Y b), por otro lado, aunque distintos autores amplían o modifican las necesidades que se presentan (Alderfer, 1972; López Sánchez, 2008), éstas podrían ser aceptadas de manera genérica o con pequeños matices. Sin embargo, lo que no se acepta es la jerarquización que se establece ni la manera en la que se activa cada una de ellas. Surgen dificultades ante algunas realidades que ponen en entredicho la propuesta. Por ejemplo: ¿qué hay del poeta hambriento?, y ¿del escalador que pone en riesgo su vida por alcanzar la cumbre? Maslow reconocía ciertos desajustes al respecto $\mathrm{y}$, por tanto, que se produjesen algunas excepciones. Pero no se trataban de simples excepciones, lo que se estaba poniendo de manifiesto era la necesidad de una reformulación al respecto.

\title{
3. Metodología de investigación
}

Como ya se ha mencionado, para localizar todos los aspectos que tuvieron una trascendencia significativa en la historia de Suleimán y examinar pedagógicamente aquel proceso, era necesario tener una visión general de todo ello. Había que prestar atención al proceso de reforma juvenil (sobre el que se ubicaba el foco de investigación), pero también a lo ocurrido 'Antes de la detención' (las condiciones de vida en su lugar origen, la inmigración irregular, inquietudes, etc.) y a lo acaecido 'Después de las medidas judiciales' (la vida que construyó tras haber saldado cuentas con el sistema de justicia). El método biográfico era el que mejor se adaptaba a las necesidades que se planteaban.

\subsection{Fuentes de información y técnicas de recogida de datos}

El método biográfico permite la utilización de diversas fuentes y técnicas de recogida de información. En esta investigación se han usado múltiples y variados medios para recopilar datos. A continuación, se relacionan los más destacados:

Se realizaron cinco entrevistas biográficas a Suleimán, siguiendo las directrices que Pujadas (2002) establece para dichas entrevistas. En dichas entrevistas se realizó un 
recorrido pormenorizado por toda su vida, prestando especial atención al proceso de reforma juvenil. En aquella narración aparecían personas que habían sido significativas. Los investigadores elaboraron una lista con todas ellas. Otro día se solicitó a Suleimán elaborar un sociograma de su caso, es decir, una relación de las personas que, a su modo de ver, habían contribuido, de algún modo, a que se materializase aquel cambio en su vida. A partir de la lista elaborada por los investigadores en un primer momento y de la información recabada a través del sociograma, y teniendo como criterio de selección la trascendencia en el caso sujeto a estudio, se concretaron los siguientes 17 personajes secundarios que también fueron entrevistados (en realidad fueron doce personas, pero algunas de ellas jugaron un doble rol, por eso se habla de personajes y se contabilizan hasta diecisiete):

- Un Hermano de Suleimán.

- La Jueza que llevó el caso.

- El Subdirector del Centro de Internamiento de Menores Infractores (CIMI).

- La Trabajadora Social del CIMI y directiva de la asociación en la que Suleimán trabaja actualmente.

- El Tutor de Suleimán en el CIMI.

- Una Educadora del CIMI y, actualmente, integrante de un Equipo Técnico.

- Dos Monitores del CIMI que en la actualidad Amigos de Suleimán.

- Una señora que trabajaba en la lavandería del CIMI que, posteriormente, se convirtió en su Madre de Acogida.

- La Tutora de Suleimán durante la Libertad Vigilada.

- Un Técnico de Medio Abierto.

- Un Compañero de trabajo, piso y amigo de Suleimán.

Todos estos informantes fueron entrevistas de manera biográfica, prestando atención a los focos de investigación establecidos. De dichas entrevistas se recopilaron un compendio de testimonios significativos que posibilitaron una comprensión completa de su vida, desde la infancia hasta el momento de las entrevistas, haciendo especial hincapié en el proceso de reforma juvenil.

Las fuentes documentales más destacadas fueron: 1) el Expediente en el Juzgado de Menores y 2) el Expediente en la Dirección General de Reforma Juvenil. En ambos expedientes, se encontró numerosa información relacionada con distintos momentos del proceso de reforma vivido. Por otro lado, se utilizó la "Ley del Menor" vigente durante el caso (LO 5/2000, reguladora de la responsabilidad penal de los menores). También se recopilaron más de 200 fotografías, la mayoría de ellas correspondientes a experiencias correspondientes a la medida de internamiento en centro. Además, se elaboraron distintos materiales y se emplearon otros instrumentos de recogida de información que completaron el trabajo de campo. A continuación, se presenta un cuadro en el que se resumen todas ellas (tabla 1). 
Tabla 1. Resumen de las fuentes de información y de las técnicas de recogida de datos.

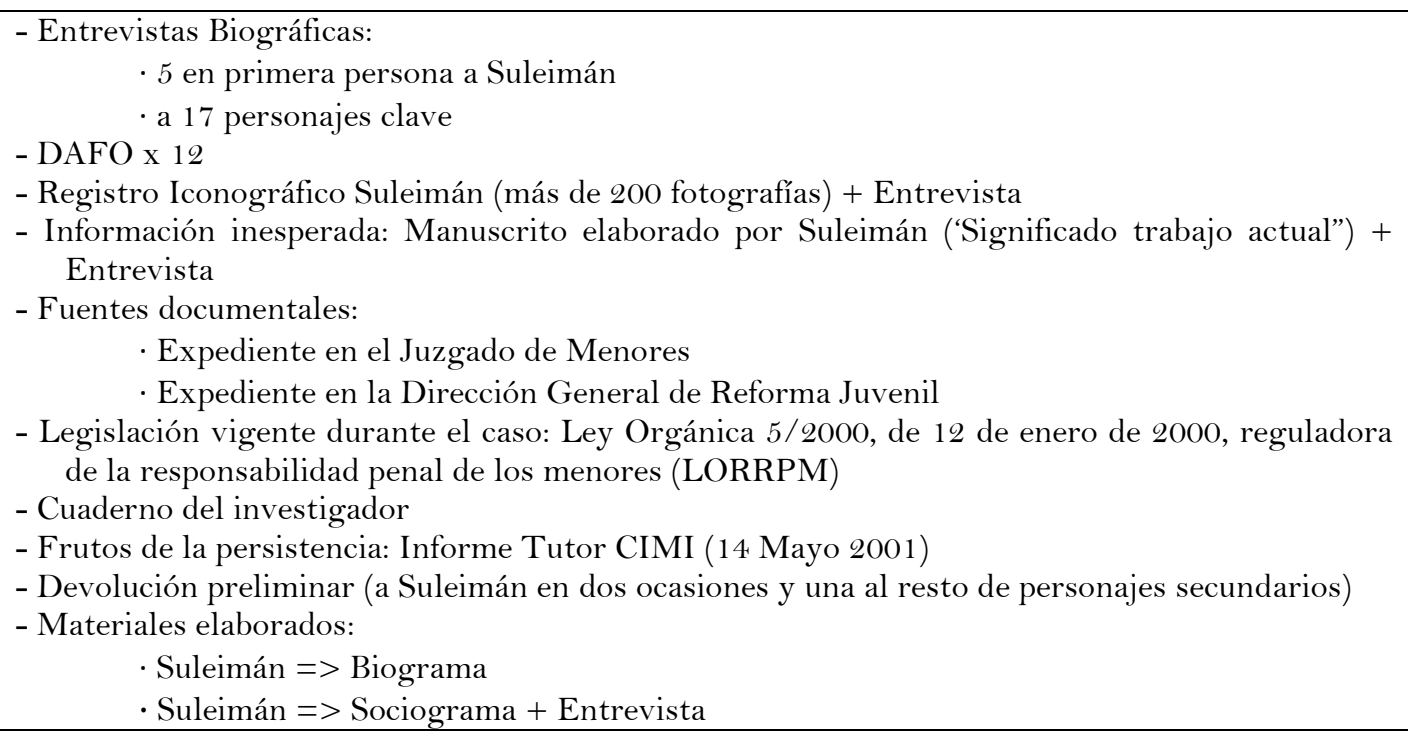

Fuente: Elaboración propia.

Como se puede ver en la tabla, la biografía de Suleimán fue construida tomando en consideración una enorme cantidad de información. La convergencia de datos provenientes de tantas fuentes y tan diversas técnicas proporcionó la oportunidad de comprender y llevar a cabo una historia de vida compleja y exhaustiva.

\section{Resultados}

Suleimán tuvo una nueva oportunidad en la vida porque, además de otros motivos, la intervención que se llevó a cabo con él a propósito del proceso de reforma juvenil se materializó de manera personalizada, es decir, atendiendo a sus características individuales y circunstanciales. Como veremos a continuación dicha personalización se articuló a través de distintas iniciativas y situaciones pedagógicas, pero también mediante la satisfacción de diversas necesidades, fundamentalmente sociales, que mediatizaban su caso. Contribuir en la satisfacción de dichas necesidades y, por tanto, personalizando la intervención, fue esencial para que su historia evolucionara de manera satisfactoria.

A continuación, se presentan las quince claves que al término de la investigación se consideraron fundamentales para que su caso diese aquel giro. De ellas, sólo cinco (que aparecen en cursiva) tuvieron carácter educativo, el resto fueron aspectos, mayoritariamente, de carácter social:

- Características personales de Suleimán.

- Interpretó el proceso de reforma como una oportunidad. Tuvo una firme determinación por cambiar su situación.

- No tenía "perfil delictivo". Tenía unas pautas de comportamiento adecuadas.

- La familia biológica fue un factor protector muy importante. 
- Religiosidad (inculcada por su familia biológica).

- Detención (punto de inflexión fundamental).

- Las características específicas del Centro de Internamiento (ambiente de seguridad y confianza) resultaron idóneas para Suleimán.

- Ubicar a Suleimán en la sección que mejor se adecuaba a sus características evitó posibles conflictos y contribuyó a su evolución.

- El tutor del Centro de Internamiento de Menores Infractores (CIMI) dotó de sentido pedagógico la intervención en el centro.

- La Formación Reglada posibilitó la obtención del Graduado Escolar, que resultó básico para la obtención de su actual trabajo.

- Disfrutó de muchos momentos de libertad durante la medida de Internamiento en Centro, lo que facilitó el desarrollo de todo el proceso.

- La familia de acogida subsanó su situación de desamparo y le proporcionó un entorno primario de referencia que facilitó todo el proceso.

- La trabajadora social del CIMI fue fundamental en su: Regularización, Acogimiento Familiar y, sobre todo, Trabajo.

- Tuvo sintonía con algunos profesionales del Cimi y configuró un círculo social con ellos. De hecho, mantiene la relación con algunos en la actualidad.

- El Trabajo le proporcionó suficiencia económica y estabilidad vital. Lo que posibilitó regularizar su situación y establecerse de manera definitiva.

Dos terceras partes de las claves que resultaron fundamentales para que la vida de Suleimán cambiase de aquella manera no fueron pedagógicos. Eso refuerza la idea de que, a la hora de educar, además de poner en marcha los procesos educativos necesarios, hay que tener en cuenta otros aspectos que requieren ser satisfechos para que las distintas situaciones que afectan a la persona evolucionen de manera favorable. Si las circunstancias mejoran, es más fácil que el sujeto progrese.

Se puede inferir, por tanto, que en el ámbito de los menores en situación de riesgo la intervención debe ser, como mínimo, socio-pedagógica, ya que la evolución del educando debe desarrollarse a partir de aprendizajes que le modifiquen individualmente, pero también de la transformación de las situaciones sociales que sean necesarias (Cfr. Pérez, 2012). La intervención debe contribuir en ambos sentidos.

En las siguientes líneas queremos presentar las cuatro claves no pedagógicas que se pusieron en marcha durante el proceso de reforma de Suleimán, que resultaron determinantes para que su historia diese aquel giro positivo. Con ello se quiere reivindicar la naturaleza social que ha de tener toda intervención pedagógica cuando se trabaje en este ámbito:

\section{La trabajadora social del centro de internamiento}

Aquella profesional fue vital en la evolución de su historia. Las acciones más destacadas que llevó a cabo con nuestro protagonista fueron: Ayudarle a regularizar su situación en España; Mediar en el acogimiento familiar; y, sobre todo, proporcionarle el trabajo que 
posee en la actualidad. Vamos a abordar la primera de ellas, el resto se irán desgranando en los siguientes epígrafes.

Desde que Suleimán ingresó en el centro de internamiento expresaba su deseo de establecerse en España de manera definitiva y regularizada: “(...) Suleimán es un chico que desea mejorar y según él manifiesta vino a España con deseos de mejorar (...)” y "Suleimán quiere residir en España, pues considera que en ésta, hay más posibilidades" según el Informe inicial de la Trabajadora Social (p. CC).

Tras aquella información la trabajadora social del Centro de Internamiento de Menores Infractores (CIMI) se puso manos a la obra para tratar de ayudarle a alcanzar aquel objetivo. En aquella época (2001-2003) concurrían una serie de circunstancias que facilitaron la realización de los trámites pertinentes para regularizar la situación del menor. Por un lado, la legislación migratoria era mucho más permeable que la actual. Y por otro, la situación oficial en la que el joven se encontraba (menor de edad, cumpliendo medidas judiciales de reforma y en situación de desamparo) le favorecía. Al tratarse de un Menor Extranjero No Acompañado (MENA) y estar sometido a internamiento en un centro de menores infractores, la administración autonómica se vio obligada a responsabilizarse de él legalmente durante aquel periodo, lo que contribuyó favorablemente en el procedimiento de regularización migratoria, pues facilitó la adquisición de distintos permisos (residencia y trabajo), la obtención del NIE (carné de identidad para extranjeros), etc. De lo contrario, si nuestro protagonista no hubiese tenido aquel respaldo, le habría resultado más difícil regularizar su situación.

Dice Suleimán que para establecerse de manera legal en España sólo había que unir las piezas y que ellos las tenían todas. La trabajadora social del CIMI fue fundamental para conseguirlas y completar aquella gestión.

Después del juicio, con "el dinero que tenía todavía por ahí, que no había gastado, se cobró el abogado que me estaba defendiendo en ese momento y otra abogada que era la que me arregló los papeles. Ella lo tuvo todo fácil, además cobró prácticamente por toda la cara. ¿̇Por qué lo tuvo fácil? Ella lo único que ha conseguido un contrato de trabajo falso, yo tenía unos buenos informes y además la tutela la tenía la Junta de Andalucía. Yo era un niño de la Junta, todo era fácil, legalmente sólo tenía que juntar las piezas. Y las tenía todas (Suleimán, Biografía, p. CXXXIII).

Cuando se acercaba la época para que Suleimán pasase a Libertad Vigilada, la trabajadora social del CIMI se desplazó con él hasta el consulado de su país, para recopilar documentación con el objetivo de inscribirlo en la oficina de empleo y buscarle una ocupación. Consiguieron el pasaporte, tenían el permiso de residencia y sacaron la tarjeta de demanda de empleo, era el momento de encontrarle un puesto de trabajo que le permitiese subsistir dignamente cuando finalizase el proceso de reforma juvenil.

Un día tuvimos que ir a Algeciras [al consulado de Marruecos] (...). Total, nos dieron nuestra documentación, nos vinimos con nuestro pasaporte. A través del pasaporte buscamos la residencia. Ya teníamos la residencia, teníamos el pasaporte, ya tenía dieciséis años, sacamos el tema del paro y ahora vamos a ver dónde te vamos a enganchar. (Trabajadora Social del CIMI, Biografía, p. CCXVI)

\section{La familia de acogida}

La situación de Suleimán resultaba compleja a la hora de materializar algunos elementos del proceso de reforma juvenil, ya que al no tener un entorno de referencia en España durante dicho proceso se hacía imposible poner en juego algunos recursos destinados al 
descanso y al favorecimiento de su integración social (por ejemplo, algunas salidas con familiares, fines de semana, etc.).

Sin embargo, durante la medida de Internamiento en Centro, nuestro protagonista estableció una relación fraternal con una señora que trabajaba en la lavandería del CIMI. La relación se afianzó tanto que tras unos meses se solicitaron distintos permisos y se realizaron una serie de trámites burocráticos para que esta señora (junto con su familia) comenzase a sacar a Suleimán de paseo algunos días. La Jueza que llevó el caso recuerda aquel episodio:

En los permisos se supone que el menor sólo puede salir con su representante legal o con educadores, como era un menor además que estaba en 'desamparo' planteaba un problema de si podría o no salir. $Y$ a mí tal y como me lo plantearon me pareció una historia muy bonita y autoricé esas salidas" con esa señora. (Biografía, p. CCX)

Para Suleimán fue una fortuna cruzarse con aquella mujer (una persona autóctona, responsable y de confianza) y que ésta quisiera responsabilizase de él de aquella manera. Cuando llegó el momento de solicitar la modificación de medida para que pasase a libertad vigilada, la situación de desamparo en la que se encontraba suponía un problema. Entre otras cosas, porque no se debía aprobar dicha modificación sin garantizar la cobertura de una serie de necesidades básicas. Ponerlo en la calle sin asegurarse de que las necesidades mínimas iban a estar cubiertas habría sido ponerlo en una situación de riesgo: casi de indigencia y muy expuesto a volver a vincularse a la organización narcotraficante.

Ante aquel panorama aquella señora tendió su mano de nuevo para que se pudiese realizar la modificación de medida con garantías. Y ofreció a nuestro protagonista la posibilidad de irse a vivir con ellos.

Aquella mujer "siempre estaba al día de cómo va mi situación. Entonces claro, cuando se acercó la fecha para salir del centro me dijo: «¿Qué vas a hacer?», y le dije que yo no quiero volver a lo que estaba antes [(a la mafia). Entonces ella me dijo] que mejor era irme con ellos. Como nos conocíamos, yo llevaba ya tiempo saliendo los fines de semana con ellos y veían que yo era lo suficiente responsable, dijeron que sí, que no hay ningún problema, que podía ir con ellos. (Suleimán, Biografía, p. CXIV)

El papel que aquella mujer jugó en la historia de Suleimán no sólo favoreció el desarrollo de la medida de internamiento en centro, además contribuyó a la evolución de todo el proceso de reforma juvenil. ¿Qué habría ocurrido si aquella señora no hubiese jugado aquel papel? El propio Suleimán insinúa en la evidencia anterior que habría vuelto a trabajar para la mafia. Y otros profesionales del CIMI pensaban que así habría sido también.

Si no hubiese sido por aquella señora este niño hubiese sido carne de cañón otra vez.

(Monitora del CIMI, Biografía, p. CCCIV)

Trabajo

La inserción laboral era uno de los objetivos prioritarios del proceso de reforma. Se pretendía que encontrase un trabajo digno que le proporcionara los ingresos necesarios para independizarse y asentarse de manera definitiva en España. En ese sentido, la suficiencia económica se consideraba un elemento fundamental de estabilidad y emancipación. 
Que nuestro protagonista encontrase un trabajo adecuado resultaba esencial para el desarrollo de su proceso de reforma. Trabajar no sólo permite al sujeto obtener un salario y posibilidades de subsistencia, sino que además estimula su desarrollo social y su adaptación a las normas de la comunidad.

La trabajadora social del CIMI también era directiva de una asociación cuya labor era materializar medidas de protección de menores. En dicha asociación había un piso tutelado con una población de niños y adolescentes de origen marroquí un tanto complicados. Era un lugar que necesitaba de un profesional que pudiera satisfacer las necesidades que allí se planteaban y parecía que Suleimán tenía un perfil que podía cubrir dichas necesidades. En virtud de ello, le ofreció un empleo allí como monitor.

Yo veía que cara a los niños él sabía perfectamente manejar situaciones difíciles, su
experiencia le servía positiva (...) Y como yo mandaba, pues hacía falta un monitor
para un centro de la asociación (...). Se lo dije a la presidenta, digo: (...) «Tengo el
caso de un chico que es marroquí, creo que va a poner orden en el piso tal», ese piso
estaba totalmente disparado. Y se lo ofrecí, digo: «No vas a ganar mucho, pero vas
a tener un trabajo» digno. (Trabajadora Social del CIMI, Biografía, p. CCCIII)

En la actualidad, más de diez años después, continúa trabajando allí (desde hace algún tiempo como educador). Aquel trabajo lo insertaba en el tejido productivo y, además, le ayudó a regularizar su situación en España de manera permanente, ya que le permitió obtener un permiso de residencia definitivo y estabilizarse legalmente.

\begin{abstract}
La trabajadora social "sabía que los papeles que a mí me han dado la primera vez eran de un año, pero para renovarlos necesito un contrato. Entonces claro, yo estaba mirando a ver cómo puedo hacer aquello y ella pues me ha buscado ese trabajo para que pueda renovar. Yo ya tenía los papeles, simplemente cuando llega la hora de la renovación de la primera residencia te exigen que estés trabajando. Si tú no tienes un contrato para la renovación, entonces tú has venido temporal a trabajar, tu contrato ya ha acabado, entonces deberías de irte. Pero si tú tienes un contrato que sea de trabajo, si te puedes seguir estando (...). Ella sabía eso y sabía que yo buscaba trabajo. Entonces claro, me ha venido bien, todo eso me ha venido seguido porque al pasar alli [a trabajar en el piso tutelado], pues ya no tuve problemas ni nada, todo lo que me han pedido de la empresa lo di y ya se ha quedado todo claro", todo regularizado de manera definitiva. (Suleimán, Biografía, pp. CXIX-CXX)
\end{abstract}

\title{
Círculo social
}

Suleimán sintonizó muy bien con algunos de los profesionales que participaron en la medida de internamiento en centro y estableció con ellos una relación cercana. En virtud de ello, se preocupó de ir reforzando y mantener dicho vínculo con quienes consideraba eran personas de confianza. Poco a poco fue configurando un círculo social sólido que se convirtió en un resorte muy importante para salir adelante.

Aquel entramado de relaciones se constituyó en un elemento que le ayudaba a afrontar las distintas amenazas que le acechaban (situación irregular, mafia, dinero fácil, drogas, etc.). En otras palabras, funcionaba como un factor de protección que, entre otras cosas, prevenía el desarrollo de conductas problemáticas. Y, también, se tornaba una red de seguridad en caso de pérdida del equilibrio. Al estilo de la que protege al funambulista en caso de caída.

Estas cuatro claves fueron capitales a la hora de transformar las circunstancias que rodeaban el caso y que, de no haberse satisfecho se habría puesto en riesgo su evolución. 


\section{Discusión}

Son pocos los estudios que se han realizado sobre menores infractores desde un enfoque socio-pedagógico, la mayoría de dichos trabajos se han realizado desde una perspectiva criminológica o jurídica (desde el derecho penal juvenil). En ese sentido, fueron pioneros los trabajos de Garrido Genovés, en solitario (2005) y acompañando a López La Torre (2005), y Diego Espuny (2000) que abordaron la intervención educativa con dichos menores. La publicación de Martínez Reguera (2007) permitió comprender la complejidad de las situaciones en las que se encuentran esos muchachos y cómo los factores de riesgo mediatizan su desarrollo. La obra de Funes Artiaga (2008) demanda un acercamiento (social, psicológico y pedagógico) adecuado a su realidad.

En relación a la temática concreta que en este artículo se aborda destaca: a) la publicación de Chamorro (2013), donde se pone de manifiesto la necesidad de atender a las características singulares de cada sujeto. b) El artículo de Melendro, Gónzalez y Rodríguez (2013), coincide con esa idea y presenta una serie de aspectos fundamentales para optimizar la eficacia de las intervenciones que coinciden con algunos de los elementos que aquí se plantean. Y en la misma línea, c) Herrera Pastor (2013) abunda sobre los elementos necesarios para la personalización de los procesos.

En el caso de Suleimán confluyeron todos esos elementos. De hecho, si lo contrastamos con la teoría de las necesidades humanas de Maslow, podemos apreciar que el proceso de reforma le ayudó a satisfacer los cinco niveles de necesidades que se establecen en dicha teoría: 1) Sus necesidades fisiológicas estuvieron cubiertas: durante la primera medida las cubría el centro de internamiento y durante la libertad vigilada la familia de acogida. 2) En sendos escenarios se encontraba fuera de peligro y amenazas. 3) Fue formando un círculo social favorable al entorno que quería construir, con algunos profesionales del CIMI y la familia de acogida. 4) Gozaba del reconocimiento por parte del equipo interdisciplinar del Centro de Internamiento y era estimado dentro de su círculo social. 5) En su horizonte estaba su deseo migratorio, que era lo que en realidad le movía desde el primer momento.

El puente que a mí me pasó a la vida real y que tenía que pasar cualquier día fue ese centro (...), alli estuve en un punto que no tuve que preocuparme de dónde dormir ni dónde comer porque lo tenía cubierto, entonces, simplemente se ha quedado en que me aclare y que coja mi camino. (Suleimán, Biografía, p. CXXXII)

Esto viene a reafirmar la idea de que: 1) hay que personalizar el proceso de intervención. Y 2) para que dicho proceso tenga mayores posibilidades de éxito resulta fundamental tratar de contribuir en la satisfacción de las distintas necesidades que plantee el caso.

\section{Conclusiones}

A continuación, se presentan las ideas más destacadas que se sacan en claro a partir del estudio realizado:

La personalización de los procesos de intervención pedagógicos que se llevan a cabo con menores en situación de riesgo, proporciona mejores condiciones para conseguir los objetivos previstos. Y, como consecuencia, se estará en mejor disposición para intentar romper con el determinismo y optimizar la búsqueda de igualdad de oportunidades para dicho colectivo. 
Para personalizar el proceso de intervención, éste se ha de articular de manera singular, integral y participativa. Singular porque cada caso es único y, por tanto, necesita ser atendido en virtud de su particularidad, prestando atención a las características específicas del educando y de las circunstancias que lo rodean.

Integral porque, además de atender al sujeto de manera contextualizada, debe ser capaz de comprender las distintas características individuales o dimensiones que lo conforman (intelectual, afectiva, social,...) e intervenir sobre las que se estimen convenientes para lograr los objetivos que se planteen. Por supuesto para ello se ha de hacer, previamente, una evaluación exhaustiva y holística del caso.

La intervención debe ser participativa en un sentido pro-activo, se trata de que el educando sienta como suyo el programa que se está llevando a cabo. Para ello, los profesionales que implementen la intervención deben hacer que el educando le encuentre sentido y utilidad para su vida, de ese modo, será más fácil que decida involucrarse vívidamente.

Por otro lado, la intervención debe contribuir en la satisfacción de las necesidades concretas que plantee cada caso. De ello dependerá, en buena medida, la evolución del caso. De hecho, desatender alguna de ellas puede obstaculizar dicha evolución. En ese sentido, es importante conocer cómo interpreta el educando cada una de las necesidades que se detecten para contribuir en su satisfacción de manera adecuada. Resulta más sencillo que el educando progrese adecuadamente en su entorno social y en su ámbito individual, si detecta que se están implementando procesos para satisfacer dichas necesidades.

Es importante recordar que, en términos generales, los profesionales no deben solucionar los problemas o las necesidades que se planteen en este tipo de casos, sino que deben ayudar al educando a que los supere por sí mismo. En otras palabras deben ser los menores quienes tomen las riendas de la transformación de su situación y de su desarrollo.

Por todo ello, se debe entender que la intervención con este colectivo tiene una naturaleza transversal, por un lado debe ser pedagógica, pero, por otro, también debe ser (como mínimo) social.

Por otro lado, en relación a la Teoría de Maslow, ciertamente, algunas necesidades resultan más básicas que otras para los individuos. No obstante, cada uno, de manera singular, es quien va estableciendo la prioridad entre las mismas. No existe una organización predeterminada al respecto. Todo lo más que se podría aceptar es que las necesidades de nivel inferior poseen una naturaleza más básica, en cuanto que entroncan con la naturaleza biológica del ser humano. Sin embargo, eso no quiere decir que, obligatoriamente, se hayan de satisfacer siempre antes que las de nivel superior.

Por otro lado, tampoco se acepta una articulación tan rígida del funcionamiento de la teoría, pues se pueden poner en marcha de manera paralela distintos mecanismos para satisfacer al mismo tiempo necesidades de distinto orden.

\section{Referencias}

Alderfer, C. P. (1972). Existence, relatedness, and Growth: Human needs in organizational settings. Nueva York, NY: Free Press. 
Alonso, H., Ruiz, P., Sánchez, J. y Oficialdegui, R. (2014). Exclusión social, medio abierto e incorporación social en la Asociación Bizitegi: Un modelo de intervención socioeducativa en programas de reinserción social desde la Pedagogía Social Comunitaria. Educación Social, 57, 48-66.

Chamorro Barranco, P.P. (2013). Evaluación e intervención del ajuste social en jóvenes en situación de riesgo y exclusión social. En D. Sánchez-Teruel y M. A. Robles-Bello (Coords.), Transformando problemas en oportunidades: Evaluación e intervención psicosocial y educativa en la infancia y adolescencia (pp. 173-197). Jaén: Universidad de Jaén.

Diego Espuny, F. (2000). La intervención con menores infractores. En M. T. Martín López (Coord.), Justicia con menores, menores infractores y menores víctimas (pp. 55-82). Cuenca: Ediciones de la Universidad de Castilla La Mancha.

Esteve, J. M. (2010). Educar: Un compromiso con la memoria. Barcelona: Octaedro.

Funes Artiaga, J. (2008). Encontremos un lugar para la infancia. Cuadernos de Pedagogía, 382, 8083.

Garrido Genovés, V. (2005). Manual de intervención educativa en readaptación social. Fundamentos de la intervención. Valencia: Tirant lo Blanch.

Guichot, V. (2013). Participación, ciudadanía activa y educación. Revista Teoría de la Educación, $25,25-47$.

Herrera Pastor, D. (2013). Menores infractores. El taller, un medio de enorme potencial pedagógico dentro de la medida de internamiento en centro. En S. Torío López, O. García Pérez, J. V. Peña Calvo y C. M. Fernández García (Coords.), La crisis social y el estado de bienestar: Las respuestas de la pedagogía social (pp. 609-614). Oviedo: Universidad de Oviedo.

Longás, J. y Riera, J. (2011). Fracaso escolar y tránsito de los jóvenes hacia la vida adulta. Razones y propuestas para apoyar la transición de la escuela al trabajo. Educación Social, 49, 145-162.

López, F. (2008). Necesidades en la infancia y en la adolescencia. Respuesta familiar, escolar y social. Madrid: Pirámide.

López La Torre, M. J. y Garrido Genovés, V. (2005). Un modelo para la prevención e intervención de la conducta antisocial. En V. Garrido y M. J. López (Coords.), Manual de intervención educativa en readaptación social. Los programas del pensamiento prosocial (pp. 11 94). Valencia: Tirant lo Blanch.

Luque, M. J. y Lalueza, J. L. (2013). Aprendizaje colaborativo en comunidades de práctica en entornos de exclusión social. Revista de Educación, 362, 402-428. doi: 10.4438/1988-592XRE-2011-362-166

Martínez Reguera, E. (2007). Cachorros de nadie. Descripción psicológica de la infancia explotada. Madrid: Popular Ed.

Maslow, A. (1975). Motivación y personalidad. Barcelona: Sagitario.

Melendro, E. M., González Olivares, A. L. y Rodríguez Bravo, A. E. (2013). Estrategias eficaces de intervención socioeducativa con adolescentes en riesgo social. Pedagogía Social. Revista Interuniversitaria, 22, 105-121. doi: 10.7179/PSRI_2013.22.02

Naranjo, C. (2007). Cambiar la educación para cambiar el mundo. Santiago: Indigo.

Pérez, C. (Coord.). (2012). La acción educativa social. Nuevos planteamientos. Madrid: Desclee de Brouwer. 
Pujadas Muñoz, J. J. (2002). El método biográfico: El uso de las historias de vida en ciencias sociales. Madrid: CIS.

Sánchez-Raya, M. A. y Alonso García, J. (2013). Educación social: Aplicación de estrategias didácticas para la infancia en riesgo y dificultad social. En C. Sánchez Romero (Coord.), Aplicación de estrategias didácticas en contextos desfavorecidos (pp. 75-95). Madrid: UNED.

Sarramona, J. (2008) Teoría de la educación. Reflexión y normativa pedagógica. Barcelona: Ariel.

Trilla, J., Gros, B., López, F. y Martín, M. J. (2011). La educación fuera de la escuela. Ámbitos no formales y educación social. Barcelona: Ariel.

Vega Fuente, A. (1994). Pedagogía de inadaptados sociales. La educación del menor inadaptado. Madrid: Narcea.

\section{Breve CV de los autores}

\section{David Herrera Pastor}

Doctorado Internacional en Ciencias de la Educación. Actualmente disfruta un Contrato Postdoctoral en la Universidad de Málaga (España), en el dpto. Teoría e Historia de la Educación y M.I.D.E. Desde hace más de una década viene investigando, desde una perspectiva pedagógica, en el ámbito social. Le interesa, particularmente, el colectivo de menores en situación de riesgo. Entre otras cosas, porque durante algunos años trabajó como Educador Social con dicho colectivo. Investigaciones subvencionadas relacionadas con el tema: 1) una IAP en un Centro de Menores Infractores. Y 2) en un barrio excluido de Málaga. Las publicaciones más destacadas que se destilan de su trayectoria son: A) El libro "Voz y Educación" que se publicó en Octaedro en 2009. Y B) El artículo: "Un acercamiento a la situación formativa de los/as educadores/as sociales" publicado en Revista de Educación en 2010. ORCID ID: 0000-0002-2198-5537. Email: dvherrera@uma.es

\section{José Manuel de Oña Cots}

Doctor en Pedagogía desde el año 2009 y Docente Universitario; con una amplia experiencia como Educador Social, centra su investigación en el ámbito de la Pedagogía Social, realizando propuestas de acompañamiento educativo con infancia y juventud y grupos familiares en contextos de exclusión. Ha realizado también aportaciones al ámbito del Desarrollo Comunitario y la evaluación de programas y proyectos socioeducativos en territorios, publicando un libro dentro de la Colección de libros de la Fundación Foessa (Año 2010) en el que se realizan propuestas concretas de trabajo en las áreas socioeducativas señaladas. Destacan publicaciones en Revistas como "Documentación Social, Revista de Estudios Sociales y de Sociología Aplicada"; "REICE. Revista Electrónica Iberoamericana sobre Calidad, Eficacia y Cambio en Educación", "Pedagogía Social, Revista Interuniversitaria" entre otras. ORCID ID: 0000-0002-17669929. Email: josecots@uma.es 